\title{
Association between the oxytocin receptor (OXTR) gene and mesolimbic responses to rewards
}

\author{
Cara R Damiano ${ }^{1 *}$, Joseph Aloi ${ }^{1}$, Kaitlyn Dunlap², Caley J Burrus ${ }^{2}$, Maya G Mosner ${ }^{1}$, Rachel V Kozink \\ Ralph Edward McLaurin ${ }^{4}$, O'Dhaniel A Mullette-Gillman ${ }^{5,6}$, Ronald McKell Carter ${ }^{3,7}$, Scott A Huettel ${ }^{3,7,8}$, \\ Francis Joseph McClernon ${ }^{3,4}$, Allison Ashley-Koch ${ }^{9}$ and Gabriel S Dichter ${ }^{1,2,10,11}$
}

\begin{abstract}
Background: There has been significant progress in identifying genes that confer risk for autism spectrum disorders (ASDs). However, the heterogeneity of symptom presentation in ASDs impedes the detection of ASD risk genes. One approach to understanding genetic influences on ASD symptom expression is to evaluate relations between variants of ASD candidate genes and neural endophenotypes in unaffected samples. Allelic variations in the oxytocin receptor (OXTR) gene confer small but significant risk for ASDs for which the underlying mechanisms may involve associations between variability in oxytocin signaling pathways and neural response to rewards. The purpose of this preliminary study was to investigate the influence of allelic variability in the OXTR gene on neural responses to monetary rewards in healthy adults using functional magnetic resonance imaging (fMRI).
\end{abstract}

Methods: The moderating effects of three single nucleotide polymorphisms (SNPs) (rs1042778, rs2268493 and rs237887) of the OXTR gene on mesolimbic responses to rewards were evaluated using a monetary incentive delay fMRI task.

Results: T homozygotes of the rs2268493 SNP demonstrated relatively decreased activation in mesolimbic reward circuitry (including the nucleus accumbens, amygdala, insula, thalamus and prefrontal cortical regions) during the anticipation of rewards but not during the outcome phase of the task. Allelic variation of the rs 1042778 and rs237887 SNPs did not moderate mesolimbic activation during either reward anticipation or outcomes.

Conclusions: This preliminary study suggests that the OXTR SNP rs2268493, which has been previously identified as an ASD risk gene, moderates mesolimbic responses during reward anticipation. Given previous findings of decreased mesolimbic activation during reward anticipation in ASD, the present results suggest that OXTR may confer ASD risk via influences on the neural systems that support reward anticipation.

Keywords: Autism spectrum disorder (ASD), Oxytocin, Oxytocin receptor, Genetics, Neuroimaging, Reward, Motivation, Mesolimbic, Functional magnetic resonance imaging (fMRI), Single nucleotide polymorphism (SNP)

\section{Background}

Autism spectrum disorders (ASDs) are highly heritable neurodevelopmental disorders [1] and there has been significant progress in identifying genes that confer increased risk for ASDs [2,3]. However, finding clear associations between genetic risk variants and ASD symptom expression has been challenging due to the highly heterogeneous nature of the disorder. One approach to address these challenges

\footnotetext{
* Correspondence: cdamiano@email.unc.edu

'Department of Psychology, University of North Carolina, CB\#3270, Davie Hall, UNC-CH, Chapel Hill, NC 27599, USA

Full list of author information is available at the end of the article
}

and improve our understanding of genetic influences on ASD symptom expression is to evaluate relations between variants of ASD candidate genes and ASD-relevant neural endophenotypes in homogeneous unaffected samples [4]. This imaging-genetics approach has the potential to elucidate genetic influences on neural endophenotypes relevant to the expression of ASD symptoms [5].

Although there are hundreds of identified ASD risk genes [6-8], the oxytocin receptor (OXTR) gene has been shown to confer a small but significant risk for
C Biomed Central 
ASDs. The influence of the OXTR gene in ASDs has been established across different ethnic populations and different study methodologies (genome-wide association studies and linkage analyses) [9-12]. There is also evidence of altered methylation of the OXTR promoter gene and reduced expression of OXTR in the postmortem brains of individuals with ASDs, providing further evidence for the role of OXTR, the oxytocin (OXT) signaling pathway and epigenetic regulation of OXTR in the etiology of ASDs [13].

OXT is a neuropeptide that has been consistently linked to affiliative and social behaviors in preclinical and human studies [14-17]. There is accumulating evidence for the role of altered OXT functioning in the etiology of social impairments in ASDs [18]: atypical levels of plasma OXT and its precursors have been found in children with ASDs $[19,20]$ and exogenous OXT administration has been shown to improve social functioning in children and adults with ASDs [21-23].

However, the potential mechanisms by which OXT influences social functioning in ASDs remain poorly understood. One possibility is via effects of the OXT signaling pathways on mesolimbic brain systems that code for the motivational relevance of social rewards $[24,25]$. There has recently been increased interest in examining the effect of motivational factors on social functioning in ASDs. The social motivation hypothesis of ASD posits that disruption of neural mechanisms supporting social motivation may constitute a primary deficit in ASDs with potential downstream effects on the development of social cognition [26,27]. This model is supported by initial neuroimaging evidence for attenuated neural responses to social rewards in mesolimbic brain regions in ASDs $[28,29]$, though there is evidence of altered mesolimbic functioning in response to non-social rewards in ASDs as well [30,31].

Brain regions that code for reward valuation, including the nucleus accumbens and amygdala, have dense OXTR expression $[32,33]$ and OXT binding in these regions influences social behaviors [34-39]. Additionally, OXT plays a key role in regulating dopaminergic activity in mesolimbic reward circuitry [40-43]. Infusion of an OXT agonist into the ventral tegmental area results in increased dopaminergic neuron firing in the ventral striatum in a manner that predicts variability in maternal behavior [41], and administration of intranasal OXT enhances mesolimbic activation in response to social rewards (for example, viewing a smiling infant face) [44,45]. Additionally, plasma levels of OXT are associated with variability in ventral and dorsal striatal activation during reciprocated cooperation, a socially rewarding experience $[45,46]$. Variants of the OXTR gene are associated with structure and function of reward-related brain regions, including the hypothalamus, insula and amygdala [47-49].
These studies suggest that OXT may stimulate mesolimbic dopamine release, which may thereby facilitate approach behaviors towards rewards [50].

Despite the central role of OXT systems for social motivation and evidence for OXT binding in mesolimbic reward-processing brain regions, no study to date has examined the effects of individual differences in OXT functioning on mesolimbic responses to rewards. The present study examined how allelic variants of the OXTR gene influence neural responses to rewards during functional magnetic resonance imaging (fMRI). To index the neural substrates of reward processing, we used a monetary incentive delay (MID) task [51] that reliably recruits reward-processing circuitry across a wide range of clinical and non-clinical populations [52-59]. This preliminary investigation addressed only responses to monetary rewards since we were primarily interested in testing the hypothesis that OXTR variants are generally associated with dopaminergic mesolimbic activation and monetary rewards are a common laboratory proxy for primary rewards that are known to activate mesolimbic brain regions robustly [60].

Three OXTR single nucleotide polymorphisms (SNPs) were examined (rs1042778, rs2268493 and rs237887), each of which has previously been linked to individual differences in social functioning and ASD risk. The rs1042778 SNP has been associated with ASD risk as well as the severity of social impairments in ASDs [10], prosocial decision-making in typical controls [61], and social-communicative abilities in individuals with ASDs and their families [62]. The rs2268493 SNP has been linked to increased risk for ASDs and increased social impairment, communicative symptoms and restricted and repetitive behaviors in ASDs $[9,10]$. Finally, the rs237887 SNP has been associated with prosocial decision-making [61] and empathy [63] in typical controls and has been associated with an increased risk of ASDs in the Japanese population [64]. Our overarching hypothesis was that OXTR alleles previously linked to ASD risk or impaired social functioning would be associated with attenuated mesolimbic activation during reward anticipation specifically. This hypothesis is based on the regulatory effects of OXT specifically on striatal dopamine [40-43], which is a central neurotransmitter that codes for the motivational salience of anticipated rewards [65].

\section{Methods}

\section{Participants}

Initially, 41 healthy adults who had participated in a prior imaging-genetics study at Duke University were contacted to participate. Of these, 36 participants completed the fMRI scan, and five of these were excluded due to unusable genetic information, resulting in a final sample of 31 participants (14 male, see Table 1 for 
Table 1 Participant characteristics

\begin{tabular}{lccc}
\hline Characteristic & Mean & Standard deviation & Range \\
\hline Age & 23.58 & 3.15 & 19 to 31 \\
Full scale IQ & 109.41 & 2.46 & 103.75 to 112.40 \\
Verbal IQ $^{\mathrm{a}}$ & 107.98 & 2.88 & 100.96 to 112.68 \\
Performance IQ $^{\mathrm{a}}$ & 109.62 & 1.36 & 106.31 to 111.84 \\
SCL-90-R GSI $^{\mathrm{b}}$ & 0.18 & 0.14 & 0.02 to 0.47 \\
AQ $^{c}$ & 13.03 & 5.85 & 2 to 26 \\
Sex & 17 female, 14 male & \\
\hline
\end{tabular}

${ }^{a}$ Estimated from the National Adult Reading Test - Revised (NART-R).

${ }^{\mathrm{b}} \mathrm{GSI}$, Global Severity Index; IQ, intelligence quotient; SCL-90-R, Symptom Checklist-90-Revised (clinical cut-off $=2.49[68]$ ). ${ }^{\mathrm{C}} \mathrm{AQ}$, autism spectrum quotient.

demographic information). Participants were right-handed, were not taking any psychotropic medications and had no current or past psychiatric disorders according to the Structured Clinical Interview for DSM-IV Axis I Disorders (SCID-I) [66], which was administered by a trained interviewer and supervised by a licensed psychologist. All participants scored below the clinical cut-off for the Symptom Checklist-90-R (SCL-90-R) [67,68], a general measure of clinical distress or psychopathology. Participants also completed the National Adult Reading Test - Revised (NART-R). Intelligence quotient (IQ) [69] and the autism spectrum quotient [70], a continuous measure of autism symptomatology, were estimated. To minimize effects of population stratification, the sample included only nonHispanic Caucasian participants. This study was reviewed and approved by the Duke University Institutional Review Board and written informed consent was obtained from all participants.

\section{Genotyping}

The Oragene DISCOVER (OGR-250) collection kit (formerly known as the Oragene DNA collection kit) (DNA Genetic Inc, Kanata, ON, Canada) was used to obtain genetic information. All participants provided at least $5 \mathrm{~mL}$ of saliva for the genetic sample. DNA was isolated using QIAGEN Autopure LS (Venlo, The Netherlands). As part of a larger study investigating a custom set of SNPs, genotyping was performed by the Center for Human Genome Variation at Duke University using a GoldenGate assay with the Illumina BeadXpress platform (Illumina, Inc, San Diego, CA, USA).

This procedure provided allelic information for the following OXTR SNPs: rs53576, rs237885, rs237887, rs237900, rs1042778, rs2254295, rs2268493, rs4686301, rs9840864 and rs11131149. The available OXTR variants with intact SNP data (call rates $>0.9$ ) for all 31 participants included four SNPs: rs237887, rs1042778, rs2254295 and rs2268493. From these four SNPs, three SNPs (rs1042778, rs2268493 and rs237887) were selected based on minor allele frequencies $>0.20$ in the present sample (the minor allele frequency for the rs2254295 SNP was 0.12). These three SNPs have also been associated with individual differences in social functioning and/or ASD risk in previous studies. For example, see $[9,10,62]$. Allelic frequencies in these SNPs were similar to the minor allele frequency expected in Caucasian samples and were consistent with Hardy-Weinberg equilibrium (rs2268493: $\chi^{2}=0.03$; rs1042778: $\chi^{2}=0.03$; rs237887: $\chi^{2}=0.05$, in the present sample).

Across all SNPs, risk allele homozygotes were compared to a combined group of non-risk allele homozygotes and heterozygotes. For rs2268493, the $\mathrm{T}$ allele has been associated with ASD risk $[9,10]$, and thus we compared 14 risk allele homozygotes (TT) to a combined group of 17 non-risk allele homozygotes and heterozygotes $(\mathrm{CC} / \mathrm{CT})$. For rs237887, the A allele has been linked to increased risk for ASD in the Japanese population [64] as well as decreased prosociality [61], and thus we compared nine risk allele homozygotes (AA) to a combined group of 22 heterozygotes and non-risk allele homozygotes (AG/GG). For rs1042778 the G allele has been linked to ASD risk [10,62], and thus we compared 14 risk allele homozygotes (GG) to 17 non-risk allele homozygotes and heterozygotes (TT/TG).

Participants included in the current study were drawn from a larger sample, which included 508 Caucasian participants. To evaluate potential linkage disequilibrium among the three OXT SNPs examined in the present study (rs1042778, rs2268493 and rs237887), we calculated linkage disequilibrium in the larger sample from which these participants were drawn. These SNPs were not in linkage disequilibrium in this larger sample, since in all cases $r^{2}<0.64$ :

- rs1042778 and rs2268493: $r^{2}=0.078$

- $r s 1042778$ and rs237887: $r^{2}=0.291$

- rs2268493 and rs237887: $r^{2}=0.211$

- rs2268493 and rs1042778: $r^{2}=0.078$

- $r$ rs237887 and rs1042778: $r^{2}=0.291$

- $r s 237887$ and rs2268493: $r^{2}=0.211$

These were consistent with linkage disequilibrium values from the HapMap website [71].

\section{fMRI task}

Participants completed two runs of a monetary incentive delay (MID) task [57] as part of a 1-hr-long fMRI scan session. During this scan session, the participants also completed two runs of an unrewarded go/no-go task. The MID task and the go/no-go task were presented in a pseudo-randomized, counterbalanced order, and only the results of the MID task are presented here.

Each MID trial consisted of: (1) a 2,000-ms cue indicating whether a monetary reward could be won (a triangle) or not (a circle) on a given trial; (2) a 2,000 to 
2,500 ms crosshair fixation; (3) a target bullseye presented for up to $500 \mathrm{~ms}$ that required a speeded button press; (4) 3,000 ms of feedback to indicate if the participant gave a sufficiently fast response and (5) a variable length inter-trial interval crosshair. The total duration of the trial was $12 \mathrm{~s}$. For trials in which a monetary reward was possible (incentive trials), a sufficiently fast response resulted in the presentation of an image representing a gain of $\$ 1$ per successful trial, whereas a slower response resulted in presentation of an ' $\mathrm{X}$ ' indicating that no money had been won. For trials in which a monetary reward was not possible (non-incentive trials), the participants were still instructed to respond as quickly as possible to the bullseye image. During these nonincentive trials, a sufficiently fast response resulted in the presentation of a checkmark symbol indicating a successful response and no monetary gain, whereas a slower response resulted in the presentation of an ' $\mathrm{X}$ ' indicating an unsuccessful response and no monetary gain. Potential reward and non-reward trials were aperiodic and pseudo-randomly ordered. Each run included 40 trials (50\% reward trials, 50\% non-reward trials). The participants were instructed to try to be successful on as many trials as possible, and that success was contingent on response times. The response time threshold for a reward trial was adaptive to individual differences in response times so that all participants were successful for approximately two-thirds of trials (that is, approximately $66.67 \%$ accuracy; actual average accuracy was $66.85 \%, \mathrm{SD}=0.04$ ). All stimuli were presented using E-Prime presentation software (Psychology Software Tools Inc, Pittsburgh, PA, USA) and were viewed through magnet-compatible goggles (Resonance Technology Inc, Northridge, CA, USA).

\section{fMRI collection and analysis}

Scanning was performed with a General Electric Health Technologies 3-T MR750 scanner (General Electric, Waukesha, WI, USA) with $50-\mathrm{mT}^{-1}$ gradients at a $200 \mathrm{~T} \cdot \mathrm{m}^{-1} \cdot \mathrm{s}^{-1}$ slew rate. Foam cushions were placed around the head to minimize motion. A 32-channel head coil was used for parallel imaging with a sparse matrix in $k$-space [72] to correct for geometric distortions without the need for an additional field mapping sequence. High-resolution structural images with 162 slices were acquired using a three-dimensional fast SPGR BRAVO pulse sequence (repetition time: $7.584 \mathrm{~ms}$; echo time: $2.936 \mathrm{~ms}$; field of view: $256 \mathrm{~mm}$; image matrix: $256 \times$ 256; voxel size: $1 \mathrm{~mm}^{3}$; flip angle: $12^{\circ}$ ) and used for coregistration with the functional data. Structural images were aligned in the near-axial plane defined by the anterior and posterior commissures. Whole-brain functional images consisted of 32 interleaved slices parallel to the anterior and posterior commissure plane using a SENSE spiral pulse sequence sensitive to blood oxygenation level dependent (BOLD) contrast (repetition time: $2000 \mathrm{~ms}$; echo time: $30 \mathrm{~ms}$; field of view: $24 \mathrm{~cm}$; image matrix: $64 \times$ 64; voxel size: $4 \mathrm{~mm}^{3}$; flip angle: $77^{\circ}$ ). All runs began with four discarded radio-frequency excitations to achieve steady-state equilibrium. No participant had greater than $3 \mathrm{~mm}$ movement in the $x, y$ or $z$ dimension.

Functional data were preprocessed using FEAT (FMRI Expert Analysis Tool) [73,74] from FMRIB's software library (FSL) (www.fmrib.ox.ac.uk/fsl). Preprocessing for all functional data involved the following steps: (1) brain extraction to remove all non-brain data [75]; (2) motion correction using MCFLIRT [76]; (3) spatial smoothing using a Gaussian kernel of full width half maximum $5 \mathrm{~mm}$; (4) mean-based intensity normalization of all volumes by the same factor and (5) high-pass temporal filtering to remove slow signal drift (60 s) [76]. Functional and structural images were co-registered in native space and normalized to a standard stereotaxic space (using Montreal Neurological Institute templates). All registrations were carried out using the FMRIB Linear Image Registration Tool (FLIRT) for linear registration (affine with 12 degrees of freedom). The same transformation matrices used for structural-tostandard transformations were then used for functionalto-standard space transformations of the co-registered functional images. Registrations were applied using an intermodal registration tool $[74,76]$ and voxel-wise temporal autocorrelation was estimated and corrected using FMRIB's Improved Linear Model [77].

Initial analyses examined task effects in the entire group to verify the activation of the hypothesized mesolimbic reward circuitry by the task. Next, the primary research questions were addressed by examining the moderating effects of allelic group on task-specific brain activation. Data from anticipation and outcome phases were analyzed separately. During the anticipation phase (the time between presentation of cues and potential reward receipt), the contrast of interest was the effect of allelic group on differences in brain activation for incentive versus non-incentive trials. During the outcome phase (during presentation of feedback), the contrast of interest was the effect of allelic groups on differences in brain activation for reward versus non-reward trials. Models were evaluated separately for each of the three SNPs examined.

A double- $\gamma$ function modeled the hemodynamic response for each event type (incentive/non-incentive trials and reward/non-reward trials) in each run. Using a fixed-effects general linear model, parameter estimates from the two runs from each participant were combined. These data were then used in a random-effects analysis to compare allelic groups, using FMRIB Local Analysis of Mixed Effects (FLAME) [75,78]. Initial whole-brain analyses examining task effects in the entire sample used 
$Z$ statistic images cluster thresholded at $Z>5.25$ with a corrected cluster significance threshold of $P<0.05$. This somewhat high $Z$ threshold was used to allow visualization of effects primarily in reward-processing regions. For the analyses involving the three individual SNPs, whole-brain analyses were conducted using $Z$ statistic images thresholded at $Z>3.0$ with a corrected cluster significance threshold of $P<.05 / 3=0.0167$. Activation localizations were based on Harvard-Oxford cortical and subcortical structural probabilistic atlases, as implemented in FSLView v3.1.8.

\section{Results}

\section{Genotypic and sex effects on demographic and behavioral data}

Potential demographic differences between allelic groups were examined for each SNP. Across all three SNPs, there were no group differences in age, sex ratios, estimated IQ (performance, verbal or full scale) or general clinical symptomatology (based on SCL-90 scores), $P>.16$. There were also no significant differences between allelic groups in response times during the scanner task, $P>.20$ (see Figure 1). In addition, there were also no significant sex differences on any of these demographic variables, $P>.06$, and there were no significant sex differences in response times, $P>.83$. The mean response time across all participants was $212.70 \mathrm{~ms}(\mathrm{SD}=31.37)$.

\section{fMRI data: anticipation phase}

\section{Entire sample}

Across the entire sample, regardless of allelic group, reward anticipation activated the bilateral nucleus accumbens as well as other reward-processing regions, including the right caudate, right amygdala, left insular cortex, right

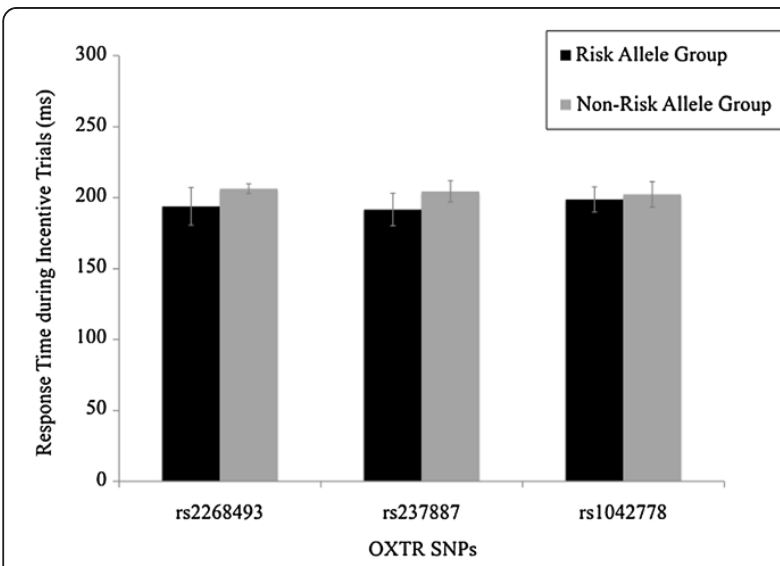

Figure 1 Response times (in ms) during incentive trials of the monetary incentive delay (MID) task for different oxytocin receptor (OXTR) gene single nucleotide polymorphisms (SNPs) grouped based on rs2268493, rs237887 and rs1042778 allelic variants. OXTR, oxytocin receptor; SNP, single nucleotide polymorphism. thalamus, right anterior cingulate gyrus and left orbital frontal cortex (see Table 2 and Figure 2). Table 2 shows activation during incentive versus non-incentive trials of the anticipation phase of the MID task across the entire sample (thresholded at $Z>5.25$, corrected cluster significance threshold of $P<.05)$.

\section{rs2268493}

During reward anticipation, activation was decreased in risk allele homozygotes (TT) relative to the combined group of heterozygotes and non-risk allele homozygotes (TC/CC) in the left nucleus accumbens, left orbital frontal cortex, right amygdala, bilateral insular cortex, left thalamus and left anterior cingulate gyrus (see Figure $3 \mathrm{a}$ and Table 3). Table 3 shows differences in brain activation between allelic groups for the rs2268493 $\mathrm{SNP}$ (that is, $\mathrm{TT}<\mathrm{CC} / \mathrm{CT}$ ) for incentive versus nonincentive trials during the anticipation phase of the MID task (thresholded at $Z>3.0$, corrected cluster significance threshold of $P<.05 / 3=0.0167)$. No regions showed relatively greater activation for the risk allele homozygotes. These general linear model analyses were then followed up with permutation testing using FSL Randomise to verify allelic differences in key regions of interest. As illustrated in Figure $3 \mathrm{~b}$, permutation testing validated the central finding of decreased activity in the left nucleus accumbens and anterior cingulate gyrus.

\section{rs237887}

Allelic variation of the rs237887 SNP (that is, risk allele homozygotes versus the combined heterozygotes and non-risk homozygotes group) did not significantly influence activation in mesolimbic regions (see Figure 4 and Table 4). Table 4 shows differences in brain activation between allelic groups for the rs237887 SNP (that is, $\mathrm{AA}<\mathrm{AG} / \mathrm{GG}$ ) for incentive versus non-incentive trials during the anticipation phase of the MID task (thresholded at $Z>3.0$, corrected cluster significance threshold of $P<.05 / 3=0.0167$ ).

\section{rs 1042778}

Allelic variation of the rs1042778 SNP (that is, risk allele homozygotes versus the combined heterozygotes and non-risk homozygotes group) did not significantly influence activation in mesolimbic regions.

\section{fMRI data: outcome phase}

Allelic variations in the rs2268493, rs237887 and rs1042778 SNPs did not moderate activation of mesolimbic regions during reward outcomes (see Additional file 1 for outcome phase results). 
Table 2 Activation during incentive versus non-incentive trials

\begin{tabular}{|c|c|c|c|c|c|}
\hline \multirow[b]{2}{*}{ Brain region } & \multirow[b]{2}{*}{ Voxels } & \multirow[b]{2}{*}{$Z$ (maximum) } & \multicolumn{3}{|c|}{ Montreal Neurological Institute coordinates } \\
\hline & & & $x$ & $y$ & $z$ \\
\hline Left accumbens & 104 & 6.60 & -10 & 10 & -8 \\
\hline Right accumbens/right caudate & 57 & 6.27 & 8 & 4 & -2 \\
\hline Right amygdala & 16 & 5.65 & 14 & 2 & -16 \\
\hline Right anterior cingulate gyrus & 693 & 6.73 & 6 & 16 & 34 \\
\hline Right brainstem & 386 & 6.72 & 8 & -28 & -18 \\
\hline Left orbital frontal cortex & 19 & 5.74 & -34 & 24 & -6 \\
\hline Left insular cortex & 82 & 6.20 & -38 & 14 & -8 \\
\hline Right intracalcarine cortex & 97 & 6.47 & 18 & -68 & 10 \\
\hline Left juxtapositional lobule cortex ${ }^{a}$ & 55 & 5.92 & -2 & 0 & 70 \\
\hline Right lateral occipital cortex ${ }^{a}$ & 12 & 6.00 & 34 & -88 & -18 \\
\hline Left lingual gyrus & 96 & 6.17 & -14 & -56 & -6 \\
\hline Left occipital pole & 67 & 6.53 & -22 & -96 & 16 \\
\hline Right precentral gyrus & 22 & 5.58 & 44 & 2 & 46 \\
\hline Left posterior cingulate & 17 & 5.78 & -2 & -24 & 26 \\
\hline Right thalamus & 266 & 7.09 & 4 & -4 & 2 \\
\hline
\end{tabular}

${ }^{\mathrm{a}}$ Two clusters within same region, coordinates and peak activation reported for highest peak activation.

\section{Discussion}

The purpose of this study was to investigate whether allelic variations of OXTR SNPs influence mesolimbic brain activation during reward processing. We found that allelic variations in rs2268493, but not rs237887 or rs1042778, were related to mesolimbic activation during reward anticipation, but not reward outcomes, in healthy adults. The regions moderated by this OXTR SNP, including the nucleus accumbens, amygdala, thalamus, insula, orbital frontal cortex and anterior cingulate gyrus, have been consistently linked to reward motivation. For reviews, see $[79,80]$. In contrast to brain activation findings, no differences between allelic groups were observed in behavioral responses during the MID task. These findings suggest a disconnect between behavioral and neural responses and that the effects of rs2268493 variations are relatively weaker at the level of behavior than at the level of brain-based endophenotypes $[81,82]$.

The association between rs2268493 and mesolimbic response during reward anticipation is consistent with preclinical evidence that the OXT and mesolimbic dopamine systems act reciprocally and become linked through early social experiences [40,42,43,83-85] and that OXT modulation may directly result in increased firing of dopaminergic neurons in the ventral striatum [41]. Additionally, in humans, exogenous OXT administration influences mesolimbic responses to social stimuli
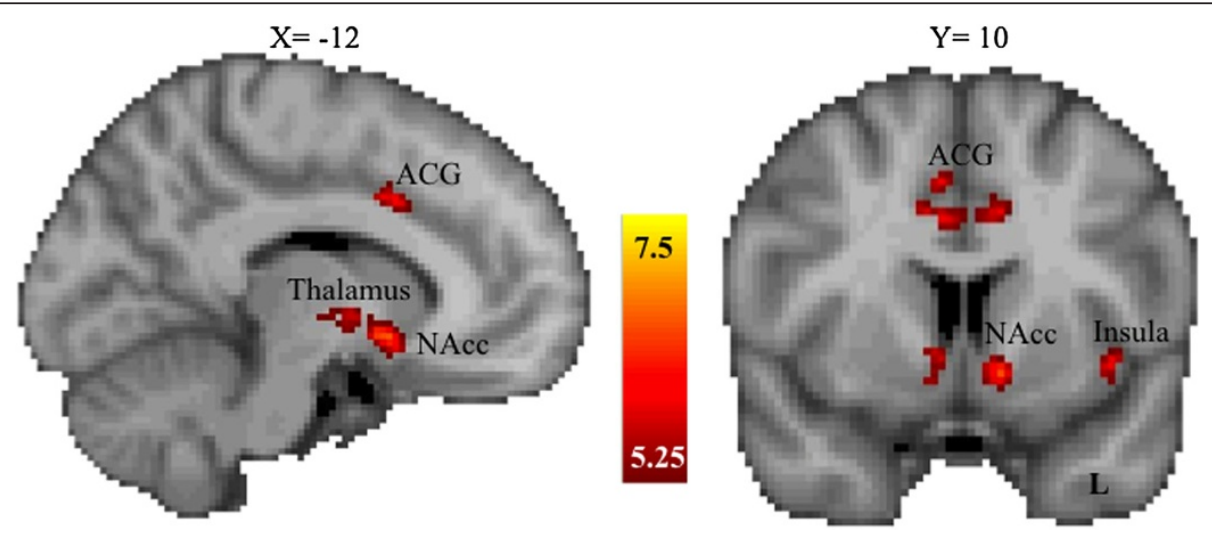

Figure 2 Brain regions showing significant activation for incentive versus non-incentive trials during the anticipation phase of the monetary incentive delay task across the entire sample. $Z$ values indicate cluster thresholds. ACG, anterior cingulate gyrus; $L$, left; NAcc, nucleus accumbens. 

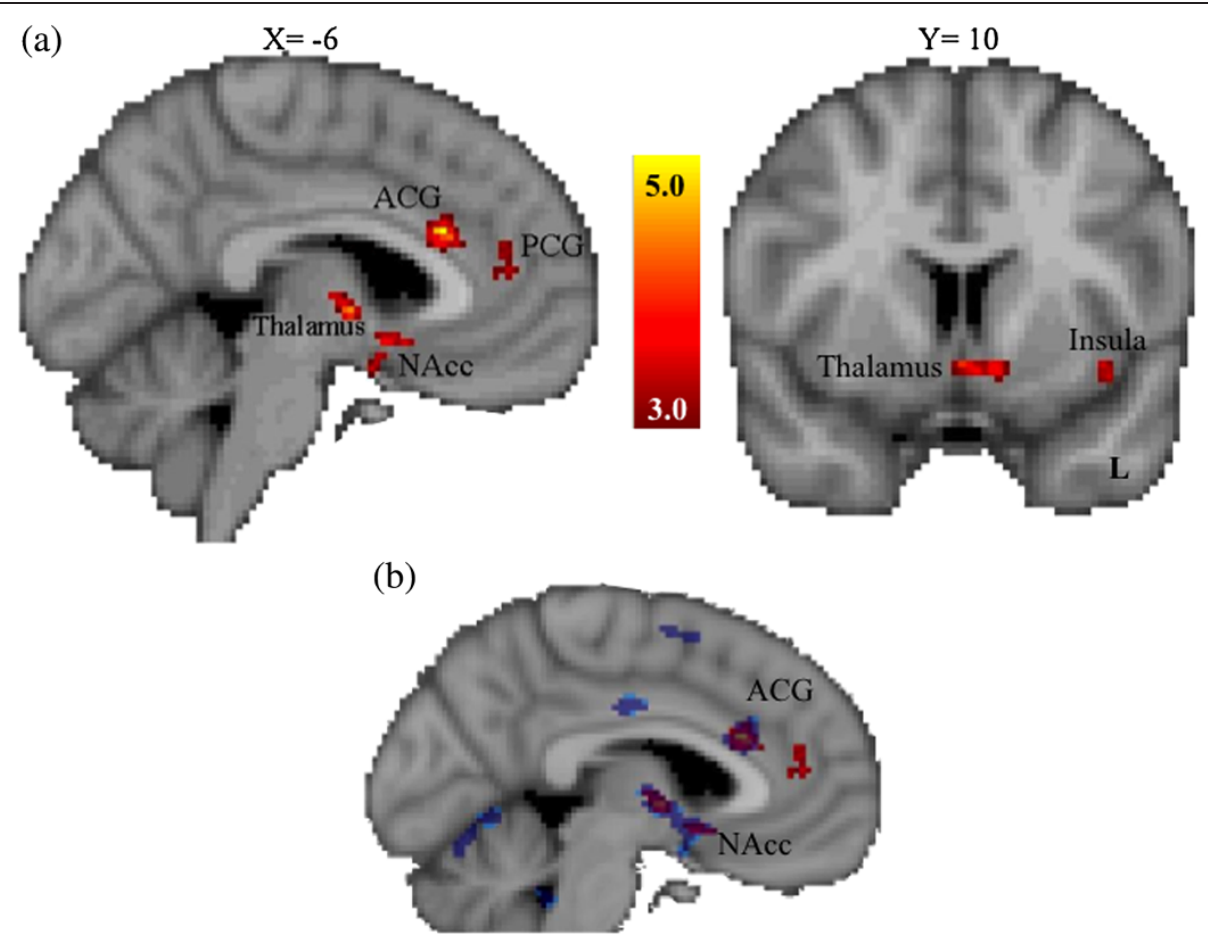

Figure 3 Brain regions showing significantly reduced activation for the OXTR SNP rs2268493 risk allele group during the anticipation phase of the monetary incentive delay task using both a general linear model approach and permutation testing. (a) General linear model analyses (in red). Z values indicate cluster thresholds. (b) Randomize permutation testing analysis (in blue). Note the convergence of effects in the left NAcc and ACG. ACG, anterior cingulate gyrus; L, left; NAcc, nucleus accumbens; OXTR, oxytocin receptor; PCG, paracingulate gyrus; SNP, single nucleotide polymorphism.

[44,45,86-91] and plasma OXT levels are associated with striatal responses to a social reward (that is, reciprocated cooperation) $[45,46]$.

Understanding the moderating effects of the OXTR gene in the general population may guide the study of genetic mechanisms underlying motivation deficits in
ASDs (cf. [92]). This approach of examining the effects of gene variants irrespective of clinical diagnosis can be informative given that risk alleles related to a particular disorder may have a more direct influence on relevant neurobiological endophenotypes than the disorder itself [81]. Thus, gaining an understanding of the effects of

Table 3 Differences in brain activation between allelic groups

\begin{tabular}{|c|c|c|c|c|c|}
\hline \multirow[b]{2}{*}{ Brain region } & \multirow[b]{2}{*}{ Voxels } & \multirow[b]{2}{*}{$Z$ (maximum) } & \multicolumn{3}{|c|}{ Montreal Neurological Institute coordinates } \\
\hline & & & $x$ & $y$ & $z$ \\
\hline Left accumbens & 83 & 4.26 & -10 & 10 & -10 \\
\hline Right amygdala & 65 & 4.03 & 12 & -4 & -18 \\
\hline Left anterior cingulate gyrus $^{a}$ & 419 & 4.93 & -4 & 24 & 24 \\
\hline Brainstem & 16 & 4.24 & 0 & -22 & -20 \\
\hline Left orbital frontal cortex ${ }^{a}$ & 10 & 3.4 & -32 & 20 & -22 \\
\hline Left insular cortex ${ }^{a}$ & 59 & 3.87 & -32 & 22 & 0 \\
\hline Right insular cortex & 108 & 3.66 & 38 & 14 & -10 \\
\hline Left middle frontal gyrus & 21 & 3.43 & -34 & 10 & 32 \\
\hline Left occipital fusiform gyrus & 147 & 4.5 & -30 & -84 & -22 \\
\hline Posterior cingulate & 11 & 3.51 & 0 & -44 & 4 \\
\hline Right superior frontal gyrus ${ }^{\mathrm{a}}$ & 41 & 3.84 & 10 & 2 & 66 \\
\hline Left thalamus & 134 & 4.58 & -6 & -4 & -2 \\
\hline
\end{tabular}

${ }^{\mathrm{a}}$ Two clusters within same region, coordinates and peak activation reported for highest peak activation. 

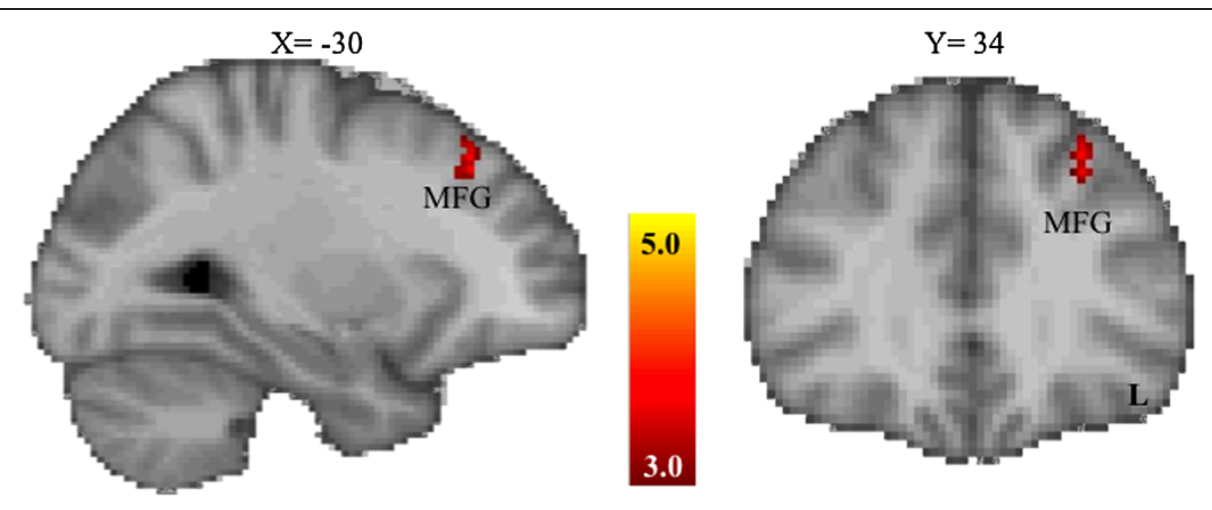

Figure 4 Brain regions showing significantly reduced activation for the OXTR SNP rs237887 risk allele group the during the anticipation phase of the monetary incentive delay task. $Z$ values indicate cluster thresholds. L, left; MFG, middle frontal gyrus; OXTR, oxytocin receptor; SNP, single nucleotide polymorphism.

risk genes in non-clinical samples may ultimately help to parse the neurobiological heterogeneity of complex neurodevelopmental disorders, such as ASDs [4].

Given the heterogeneity of ASDs and given that hundreds of different genes may be implicated in ASDs with each contributing small degrees of risk [2,92-94], it is important to emphasize that the clinical generalizability of the present findings is contingent on future studies examining the moderating influence of OXT on the mesolimbic response to rewards in individuals with ASDs. In addition, it is important to note that a comprehensive account of candidate molecular genetic influences on brain function in ASDs will require a systems-level approach that accounts for gene-gene interactions, the influence of epistasis and epigenetics on multiple neural networks and the pleiotropic effects of genes on neural phenotypes [95]. Additionally, the present study examined only mesolimbic responses to monetary rewards whereas ASDs have been associated with atypicalities in the processing of both social and non-social rewards [96]. Further research will be necessary to address OXT modulation of mesolimbic responses to social rewards, as suggested by alternative models of oxytocinergic function (for example, $[97,98]$ ), and to compare directly the effects of OXTR genes on social and non-social reward motivation in ASDs.

We also note that caution is warranted when interpreting null findings during the outcome phase of the task given the use of a recessive genetic model (that is, comparing risk allele homozygotes with other participants). This model was chosen because it is a more conservative test of the effects of the risk allele on the neural endophenotype and because our sample did not have sufficient numbers of non-risk allele carriers for meaningful analyses that compared risk allele carriers versus non-risk homozygotes (for rs2268493, only two participants were not T-allele carriers).

An important limitation of the present study was the relatively modest sample size, particularly in light of a number of recent non-replications of neuroimaging genetics studies [99]. Thus it may be that modestly sized samples in the context of candidate-gene studies are underpowered [100]. Further, a recent meta-analysis failed to confirm relations between OXTR SNPS rs53576 and

Table 4 Differences in brain activation between allelic groups

\begin{tabular}{|c|c|c|c|c|c|}
\hline \multirow[b]{2}{*}{ Brain region } & \multirow[b]{2}{*}{ Voxels } & \multirow[b]{2}{*}{$Z$ (maximum) } & \multicolumn{3}{|c|}{ Montreal Neurological Institute coordinates } \\
\hline & & & $x$ & $y$ & $z$ \\
\hline Left inferior frontal gyrus (pars opercularis) & 27 & 3.47 & -54 & 14 & 10 \\
\hline Right lateral occipital cortex & 27 & 3.74 & 48 & -72 & -2 \\
\hline Right lateral occipital pole & 12 & 3.36 & 36 & -84 & 16 \\
\hline Right lingual gyrus & 20 & 3.79 & 8 & -86 & -12 \\
\hline Left middle frontal gyrus ${ }^{a}$ & 39 & 3.81 & -28 & 32 & 44 \\
\hline Right occipital fusiform gyrus & 68 & 3.6 & 14 & -78 & -14 \\
\hline Right occipital pole & 65 & 4.44 & 12 & -94 & 16 \\
\hline Right precuneus & 36 & 3.92 & 6 & -56 & 40 \\
\hline Left supramarginal gyrus (posterior) & 60 & 3.55 & -50 & -42 & 46 \\
\hline
\end{tabular}

${ }^{\mathrm{a}}$ Two clusters within same region, coordinates and peak activation reported for highest peak activation. 
rs2254298 and measures of sociability [101]. Accordingly, replication will be necessary to draw firm conclusions about the role of rs2268493 in reward motivation. However, consistent with recommendations for imaging genetics studies [102], we used a well-validated fMRI task that had previously shown robust effects and significant variance in the typical population [103] and participants in the present study were homogeneous in terms of racial and ethnic background, age, IQ and mental health status. Although this sampling strategy limits the generalizability of results, it helps to control for the effects of these nongenetic factors [102]. It is also important to note that OXTR may not be directly related to reward motivation, but rather this relation may be mediated by other functions of the OXT system, such as anxiolytic effects (for example, [38,104]) or effects on memory (for example, [105]). Additionally, the activation clusters associated with allelic differences in rs2268493 were not confined to mesolimbic regions but rather included several regions that are not commonly associated with reward motivation (for example, the brainstem and superior frontal gyrus). Although such findings, which were not hypothesized, indicate that the moderating effects of rs2268493 may not be constrained to mesolimbic regions, future studies will be needed to replicate these results.

\section{Conclusions}

Despite these limitations and interpretive cautions, this study is a preliminary step towards a better understanding of the potential genetic mechanism underlying individual differences in neural activation during reward motivation, which may ultimately contribute to our understanding of dysfunctional motivational systems in ASDs. Although identifying brain functions associated with specific genes is an important early step in understanding the effects of particular ASD risk genes on ASD symptom expression, the link between a single gene and brain function will ultimately only explain a small part of the complex genetic heterogeneity of ASDs [97]. A systems approach to studying genetic and brain imaging data will be necessary for integrating information about the effects of multiple alleles on multiple genes, multigenic interactions on neural networks, pleiotropic effects of genes on cognitive, affective, and motor development, and the influence of environmental and epigenetic factors on these processes.

\section{Additional file}

Additional file 1: fMRI results from the outcome phase of the monetary incentive delay task. This document details the results of analyses involving brain activation during the outcome phase of the monetary incentive delay task for the entire sample and each of the three SNPs (rs2268493, rs1042778 and rs237887). Tables displaying the regions with significant activation during the outcome phase for the entire sample and for the SNP rs237887 are also included.

\section{Abbreviations}

AQ: autism spectrum quotient; ASD: autism spectrum disorder; BOLD: blood oxygen level dependent; BRAVO: brain volume imaging; DSM-V: Diagnostic and Statistical Manual of Mental Disorders (Fifth Edition); FEAT: FMRI expert analysis tool; FLAME: FMRIB's Local Analysis of Mixed Effects; FLIRT: FMRIB Linear Image Registration Tool; fMRI: functional magnetic resonance imaging;

FMRIB: functional magnetic resonance imaging of the brain; FSL: FMRIB software library; IQ: intelligence quotient; MCFLIRT: motion correction and FMRIB's linear image registration tool; MID: monetary incentive delay; NART-R: National Adult Reading Test - Revised; OXT: oxytocin; OXTR: oxytocin receptor; SCID- I: Structured Clinical Interview for DSM-IV Axis I Disorders; SCL-90-R: Symptom Checklist-90-Revised; SENSE: sensitivity encoding; SNP: single nucleotide polymorphism; SPGR: spoiled gradient echo.

\section{Competing interests}

The authors declare that they have no competing interests.

\section{Authors' contributions}

CRD coordinated the study, screened participants, collected data, analyzed behavioral and fMRI data, and drafted the manuscript. JA was involved in the collection and analysis of data and helped to draft the manuscript. KD, CJB, RVK and REM were involved in participant recruitment, collection of data and revising the manuscript. MGM, AAK, OAM, RMC and SAH were involved in the interpretation of data and drafting the manuscript. FJM helped to design and coordinate the study, interpret the data and draft the manuscript. GSD conceived, designed and coordinated the study, and helped to analyze the data and draft the manuscript. All authors read and approved the final manuscript.

\section{Acknowledgements}

This research was supported by U54 HD079124 (GSD), K23 MH081285 (GSD), RC1 MH88680 (SAH), NUS Grants R-581-000-123-133 and R-581-000-133-112 (OAM), the Weatherstone Predoctoral Fellowship (CRD), the Earl and Barbara Baughman Dissertation Research Award (CRD), and a UNC-CH Graduate School Dissertation Completion Fellowship (CRD). We would like to thank MRI technologists Susan Music, Natalie Goutkin, and Luke Poole for assistance with data acquisition, and BIAC Director Dr Allen Song for assistance with various aspects of this project.

\section{Author details}

1Department of Psychology, University of North Carolina, CB\#3270, Davie Hall, UNC-CH, Chapel Hill, NC 27599, USA. ${ }^{2}$ Brain Imaging and Analysis Center (BIAC), Duke University, Durham, NC, USA. ${ }^{3}$ Department of Psychiatry and Behavioral Sciences, Duke University, Durham, NC, USA. ${ }^{4}$ Department of Neurobiology, Duke University, Durham, NC, USA. ${ }^{5}$ Neuroscience and Behavioral Disorders Program, Duke-NUS Graduate Medical School, Singapore. ${ }^{6}$ Psychology Department, National University of Singapore, Singapore. ${ }^{7}$ Center for Cognitive Neuroscience, Duke University, Durham, NC, USA. ${ }^{8}$ Departments of Psychology and Neuroscience, Duke University, Durham, NC, USA. ${ }^{9}$ Department of Medicine, Center for Human Genetics, Duke University, Durham, NC, USA. ${ }^{10}$ Department of Psychiatry, University of North Carolina, Chapel Hill, NC, USA. ${ }^{11}$ Carolina Institute for Developmental Disabilities, University of North Carolina, Chapel Hill, NC, USA.

Received: 14 August 2013 Accepted: 17 January 2014

Published: 31 January 2014

\section{References}

1. Abrahams BS, Geschwind DH: Advances in autism genetics: on the threshold of a new neurobiology. Nat Rev Genet 2008, 9:341-355.

2. Geschwind DH: Genetics of autism spectrum disorders. Trends Cogn Sci 2011, 15:409-416.

3. State MW, Levitt P: The conundrums of understanding genetic risks for autism spectrum disorders. Nat Neurosci 2011, 14:1499-1506.

4. Rudie JD, Hernandez LM, Brown JA, Beck-Pancer D, Colich NL, Gorrindo P, Thompson PM, Geschwind DH, Bookheimer SY, Levitt P, Dapretto M: Autism-associated promoter variant in MET impacts functional and structural brain networks. Neuron 2012, 75:904-915. 
5. Munoz KE, Hyde LW, Hariri AR: Imaging genetics. J Am Acad Child AdolesC Psychiatr 2009, 48:356-361.

6. Buxbaum JD, Betancur C, Bozdagi O, Dorr NP, Elder GA, Hof PR: Optimizing the phenotyping of rodent ASD models: enrichment analysis of mouse and human neurobiological phenotypes associated with high-risk autism genes identifies morphological, electrophysiological, neurological, and behavioral features. Mol Autism 2012, 3:1-8.

7. Weiss LA, Arking DE, Daly MJ, Chakravarti A: A genome-wide linkage and association scan reveals novel loci for autism. Nature 2009, 461:802-808.

8. Anney R, Klei L, Pinto D, Regan R, Conroy J, Magalhaes TR, Correia C, Abrahams BS, Sykes N, Pagnamenta AT: A genome-wide scan for common alleles affecting risk for autism. Hum Mol Genet 2010, 19:4072-4082.

9. Yrigollen CM, Han SS, Kochetkova A, Babitz T, Chang JT, Volkmar FR, Leckman JF, Grigorenko EL: Genes controlling affiliative behavior as candidate genes for autism. Biol Psychiatry 2008, 63:911-916.

10. Campbell DB, Datta D, Jones ST, Batey Lee E, Sutcliffe JS, Hammock EAD Levitt P: Association of oxytocin receptor (OXTR) gene variants with multiple phenotype domains of autism spectrum disorder. J Neurodev Disord 2011, 3:101-112.

11. Ylisaukko-oja T, Alarcón M, Cantor RM, Auranen M, Vanhala R, Kempas E, von Wendt $L$, Järvelä I, Geschwind DH, Peltonen L: Search for autism loci by combined analysis of Autism Genetic Resource Exchange and Finnish families. Ann Neurol 2006, 59:145-155

12. Wu S, Jia M, Ruan Y, Liu J, Guo Y, Shuang M, Gong X, Zhang Y, Yang X, Zhang D: Positive association of the oxytocin receptor gene (OXTR) with autism in the Chinese Han population. Biol Psychiatry 2005, 58:74-77.

13. Gregory S, Connelly J, Towers A, Johnson J, Biscocho D, Markunas C, Lintas C, Abramson R, Wright $H$, Ellis P: Genomic and epigenetic evidence for oxytocin receptor deficiency in autism. BMC Med 2009, 7:62.

14. Insel TR, Hulihan TJ: A gender-specific mechanism for pair bonding: oxytocin and partner preference formation in monogamous voles. Behav Neurosci 1995, 109:782-789.

15. Domes G, Heinrichs M, Michel A, Berger C, Herpertz SC: Oxytocin improves 'mind-reading' in humans. Biol Psychiatry 2007, 61:731-733.

16. Guastella AJ, Mitchell PB, Dadds MR: Oxytocin increases gaze to the eye region of human faces. Biol Psychiatry 2008, 63:3-5.

17. Chen FS, Barth ME, Johnson SL, Gotlib IH, Johnson SC: Oxytocin receptor (OXTR) polymorphisms and attachment in human infants. Front Psychol 2011, 2:1-6.

18. Insel TR: The challenge of translation in social neuroscience: a review of oxytocin, vasopressin, and affiliative behavior. Neuron 2010, 65:768-779.

19. Modahl C, Green LA, Fein D, Morris M, Waterhouse L, Feinstein C, Levin H: Plasma oxytocin levels in autistic children. Biol Psychiatry 1998, 43:270-277.

20. Green LA, Fein D, Modahl C, Feinstein C, Waterhouse L, Morris M: Oxytocin and autistic disorder: alterations in peptide forms. Biol Psychiatry 2001, 50:609-613.

21. Hollander E, Bartz J, Chaplin W, Phillips A, Sumner J, Soorya L, Anagnostou E, Wasserman S: Oxytocin increases retention of social cognition in autism. Biol Psychiatry 2007, 61:498-503.

22. Andari E, Duhamel JR, Zalla T, Herbrecht E, Leboyer M, Sirigu A: Promoting social behavior with oxytocin in high-functioning autism spectrum disorders. Proc Natl Acad Sci 2010, 107:4389-4394.

23. Guastella AJ, Einfeld SL, Gray KM, Rinehart NJ, Tonge BJ, Lambert TJ, Hickie IB: Intranasal oxytocin improves emotion recognition for youth with autism spectrum disorders. Biol Psychiatry 2010, 67:692-694.

24. Kemp AH, Guastella AJ: Oxytocin: prosocial behavior, social salience, or approach-related behavior? Biol Psychiatry 2010, 67:33-34.

25. Insel TR: Is social attachment an addictive disorder? Physiol Behav 2003, 79:351-357.

26. Dawson G, Webb SJ, McPartland J: Understanding the nature of face processing impairment in autism: insights from behavioral and electrophysiological studies. Dev Neuropsychol 2005, 27:403-424.

27. Chevallier C, Kohls G, Troiani V, Brodkin ES, Schultz RT: The social motivation theory of autism. Trends Cogn Sci 2012, 16:231-239.

28. Scott-Van Zeeland AA, Dapretto M, Ghahremani DG, Poldrack RA, Bookheimer SY: Reward processing in autism. Autism Res 2010, 3:53-67.
29. Dichter GS, Richey JA, Rittenberg AM, Sabatino A, Bodfish JW: Reward circuitry function in autism during face anticipation and outcomes. J Autism Dev Disord 2012, 42:147-160.

30. Dichter GS, Felder JN, Green SR, Rittenberg AM, Sasson NJ, Bodfish JW: Reward circuitry function in autism spectrum disorders. Soc Cogn Affect Neurosci 2012, 7:160-172.

31. Cascio CJ, Foss-Feig JH, Heacock JL, Newsom CR, Cowan RL, Benningfield MM, Rogers BP, Cao A: Response of neural reward regions to food cues in autism spectrum disorders. J Neurodev Disord 2012, 4:9.

32. Insel TR, Shapiro LE: Oxytocin receptor distribution reflects social organization in monogamous and polygamous voles. Proc Natl Acad Sci 1992, 89:5981-5985.

33. Schorscher-Petcu A, Dupré A, Tribollet E: Distribution of vasopressin and oxytocin binding sites in the brain and upper spinal cord of the common marmoset. Neurosci Lett 2009, 461:217-222.

34. Ross HE, Freeman SM, Spiegel LL, Ren X, Terwilliger EF, Young LJ: Variation in oxytocin receptor density in the nucleus accumbens has differential effects on affiliative behaviors in monogamous and polygamous voles. J Neurosci 2009, 29:1312-1318.

35. Ferguson JN, Young LJ, Insel TR: The neuroendocrine basis of social recognition. Front Neuroendocrinol 2002, 23:200-224.

36. Olazabal DE, Young LJ: Species and individual differences in juvenile female alloparental care are associated with oxytocin receptor density in the striatum and the lateral septum. Horm Behav 2006, 49:681-687.

37. Olazabal DE, Young $L$ : Oxytocin receptors in the nucleus accumbens facilitate 'spontaneous' maternal behavior in adult female prairie voles. Neuroscience 2006, 141:559-568.

38. Ferguson JN, Aldag JM, Insel TR, Young LJ: Oxytocin in the medial amygdala is essential for social recognition in the mouse. J Neurosci 2001, 21:8278-8285.

39. Ross HE, Cole CD, Smith Y, Neumann ID, Landgraf R, Murphy AZ, Young LJ: Characterization of the oxytocin system regulating affiliative behavior in female prairie voles. Neuroscience 2009, 162:892-903.

40. Melis MR, Melis T, Cocco C, Succu S, Sanna F, Pillolla G, Boi A, Ferri GL, Argiolas A: Oxytocin injected into the ventral tegmental area induces penile erection and increases extracellular dopamine in the nucleus accumbens and paraventricular nucleus of the hypothalamus of male rats. Eur J Neurosci 2007, 26:1026-1035.

41. Shahrokh DK, Zhang T-Y, Diorio J, Gratton A, Meaney MJ: Oxytocin-dopamine interactions mediate variations in maternal behavior in the rat. Endocrinology 2010, 151:2276-2286.

42. Pedersen CA, Caldwell JD, Walker C, Ayers G, Mason GA: Oxytocin activates the postpartum onset of rat maternal behavior in the ventral tegmental and medial preoptic areas. Behav Neurosci 1994, 108:1163-1171.

43. Liu Y, Wang ZX: Nucleus accumbens oxytocin and dopamine interact to regulate pair bond formation in female prairie voles. Neuroscience 2003, 121:537-544.

44. Groppe SE, Gossen A, Rademacher L, Hahn A, Westphal L, Gründer G, Spreckelmeyer KN: Oxytocin influences processing of socially relevant cues in the ventral tegmental area of the human brain. Biol Psychiatry 2013, 74:172-179.

45. Rilling JK, DeMarco AC, Hackett PD, Thompson R, Ditzen B, Patel R, Pagnoni G: Effects of intranasal oxytocin and vasopressin on cooperative behavior and associated brain activity in men. Psychoneuroendocrinology 2012, 37:447-461.

46. Strathearn L, Fonagy P, Amico J, Montague PR: Adult attachment predicts maternal brain and oxytocin response to infant cues. Neuropsychopharmacology 2009, 34:2655-2666.

47. Furman DJ, Chen MC, Gotlib IH: Variant in oxytocin receptor gene is associated with amygdala volume. Psychoneuroendocrinology 2011 36:891-897.

48. Inoue H, Yamasue H, Tochigi M, Abe O, Liu X, Kawamura Y, Takei K, Suga M, Yamada $\mathrm{H}$, Rogers MA: Association between the oxytocin receptor gene and amygdalar volume in healthy adults. Biol Psychiatry 2010, 68:1066-1072.

49. Wang J, Qin W, Liu B, Wang D, Zhang Y, Jiang T, Yu C: Variant in OXTR gene and functional connectivity of the hypothalamus in normal subjects. Neuroimage 2013, 81:199-204.

50. Champagne FA, Chretien P, Stevenson CW, Zhang TY, Gratton A, Meaney MJ: Variations in nucleus accumbens dopamine associated with individual differences in maternal behavior in the rat. J Neurosci 2004, 24:4113-4123. 
51. Knutson B, Westdorp A, Kaiser E, Hommer D: FMRI visualization of brain activity during a monetary incentive delay task. Neuroimage 2000, 12:20-27.

52. Smoski MJ, Felder J, Bizzell J, Green SR, Ernst M, Lynch TR, Dichter GS: fMRI of alterations in reward selection, anticipation, and feedback in major depressive disorder. J Affect Disord 2009, 118:69-78.

53. Juckel $G$, Schlagenhauf $F$, Koslowski M, Wüstenberg T, Villringer A, Knutson B, Wrase J, Heinz A: Dysfunction of ventral striatal reward prediction in schizophrenia. Neuroimage 2006, 29:409-416.

54. Abler B, Greenhouse I, Ongur D, Walter H, Heckers S: Abnormal reward system activation in mania. Neuropsychopharmacology 2008, 33:2217-2277.

55. Bjork JM, Smith AR, Chen G, Hommer D, Lauwereyns J: Adolescents, adults and rewards: comparing motivational neurocircuitry recruitment using fMRI. PloS One 2010, 51:827-837.

56. Breiter HC, Aharon I, Kahneman D, Dale A, Shizgal P: Functional imaging of neural responses to expectancy and experience of monetary gains and losses. Neuron 2001, 30:619-639.

57. Knutson B, Adams CM, Fong GW, Hommer D: Anticipation of increasing monetary reward selectively recruits nucleus accumbens. J Neurosci 2001, 21:1-5.

58. Figee $M$, Vink $M$, de Geus F, Vulink N, Veltman DJ, Westenberg H, Denys D: Dysfunctional reward circuitry in obsessive-compulsive disorder. Biol Psychiatry 2011, 69:867-874

59. Bjork JM, Knutson B, Fong GW, Caggiano DM, Bennett SM, Hommer D: Incentive-elicited brain activation in adolescents: similarities and differences from young adults. J Neurosci 2004, 24:1793-1802.

60. Knutson B, Cooper JC: Functional magnetic resonance imaging of reward prediction. Curr Opin Neurol 2005, 18:411-417.

61. Israel S, Lerer E, Shalev I, Uzefovsky F, Riebold M, Laiba E, Bachner-Melman R, Maril A, Bornstein G, Knafo A: The oxytocin receptor (OXTR) contributes to prosocial fund allocations in the dictator game and the social value orientations task. PLoS One 2009, 4:e5535.

62. Lerer E, Levi S, Salomon S, Darvasi A, Yirmiya N, Ebstein RP: Association between the oxytocin receptor (OXTR) gene and autism: relationship to Vineland Adaptive Behavior Scales and cognition. Mol Psychiatry 2007, 13:980-988.

63. $\mathrm{WU}$ N, Li Z, SU Y: The association between oxytocin receptor gene polymorphism (OXTR) and trait empathy. J Affect Disord 2012, 138:468-472.

64. Liu X, Kawamura Y, Shimada T, Otowa T, Koishi S, Sugiyama T, Nishida H, Hashimoto O, Nakagami R, Tochigi M: Association of the oxytocin receptor (OXTR) gene polymorphisms with autism spectrum disorder (ASD) in the Japanese population. J Hum Genet 2010, 55:137-141.

65. Berridge KC: Motivation concepts in behavioral neuroscience. Physiol Behav 2004, 81:179-209.

66. First MB: Structured Clinical Interview for DSM-IV Axis I Disorders: SCID - I: Clinician Version: Administration Booklet. Washington, DC: American Psychiatric Press; 1997.

67. Derogatis LR: SCL-90-R: Symptom Checklist-90-R: Administration, Scoring, and Procedures Manual. 3rd edition. Minneapolis, MN: National Computer Systems; 1994.

68. Groenman N, Rober I, Reitsma B, Tuymelaar Koldenhof C, Lousberg R: Patiënten met chronische benigne pijn: nader psychometrisch onderzoek met de SCL-90. [Patients with chronic benign pain: further psychometric research with the SCL-90]. NVB Pijnbulletin 1993, 13:9-12.

69. Blair JR, Spreen O: Predicting premorbid IQ: a revision of the national adult reading test. Clin Neuropsychol 1989, 3:129-136.

70. Baron-Cohen S, Wheelwright S, Skinner R, Martin J, Clubley E: The autism-spectrum quotient (AQ): evidence from Asperger syndrome/ high-functioning autism, males and females, scientists and mathematicians. J Autism Dev Disord 2001, 31:5-17.

71. International Haplotype Map Project. http://www.hapmap.org

72. Liu C, Bammer R, Moseley ME: Parallel imaging reconstruction for arbitrary trajectories using $k$ space sparse matrices (kSPA). Magn Reson Med 2007, 58:1171-1181.

73. Woolrich MW, Jbabdi S, Patenaude B, Chappell M, Makni S, Behrens T, Beckmann C, Jenkinson M, Smith SM: Bayesian analysis of neuroimaging data in FSL. Neuroimage 2009, 45:173-186.

74. Smith SM, Jenkinson M, Woolrich MW, Beckmann CF, Behrens TEJ, Johansen-Berg H, Bannister PR, De Luca M, Drobnjak I, Flitney DE:
Advances in functional and structural MR image analysis and implementation as FSL. Neuroimage 2004, 23:208-219.

75. Smith SM: Fast robust automated brain extraction. Hum Brain Mapp 2002, 17:143-155.

76. Jenkinson M, Bannister P, Brady M, Smith SM: Improved optimization for the robust and accurate linear registration and motion correction of brain images. Neuroimage 2002, 17:825-841.

77. Jenkinson M, Smith SM: A global optimisation method for robust affine registration of brain images. Med Image Anal 2001, 5:143-156.

78. Woolrich MW, Behrens TE, Beckmann CF, Jenkinson M, Smith SM: Multilevel linear modelling for FMRI group analysis using Bayesian inference. Neuroimage 2004, 21:1732-1747.

79. Bartra O, McGuire JT, Kable JW: The valuation system: a coordinate-based meta-analysis of BOLD fMRI experiments examining neural correlates of subjective value. Neuroimage 2013, 76:412-427.

80. Berridge KC, Robinson TE: Parsing reward. Trends Neurosci 2003, 26:507-513.

81. Gottesman II, Gould TD: The endophenotype concept in psychiatry: etymology and strategic intentions. Am J Psychiatr 2003, 160:636-636.

82. Siebner HR, Callicott JH, Sommer T, Mattay VS: From the genome to the phenome and back: linking genes with human brain function and structure using genetically informed neuroimaging. Neuroscience 2009, $164: 1-6$

83. Hammock EA, Levitt P: The discipline of neurobehavioral development: the emerging interface of processes that build circuits and skills. Hum Dev 2006, 49:294-309.

84. Smeltzer MD, Curtis JT, Aragona BJ, Wang Z: Dopamine, oxytocin, and vasopressin receptor binding in the medial prefrontal cortex of monogamous and promiscuous voles. Neurosci Lett 2006, 394:146-151.

85. Baskerville TA, Douglas AJ: Dopamine and oxytocin interactions underlying behaviors: potential contributions to behavioral disorders. CNS Neurosci Ther 2010, 16:e92-e123.

86. Gamer M, Zurowski B, Büchel C: Different amygdala subregions mediate valence-related and attentional effects of oxytocin in humans. Proc Nat/ Acad Sci 2010, 107:9400-9405.

87. Riem MME, Bakermans-Kranenburg MJ, Pieper S, Tops M, Boksem MAS, Vermeiren RRJM, van IJzendoorn MH, Rombouts SARB: Oxytocin modulates amygdala, insula, and inferior frontal gyrus responses to infant crying: a randomized controlled trial. Biol Psychiatry 2011, 70:291-297.

88. Baumgartner T, Heinrichs M, Vonlanthen A, Fischbacher U, Fehr E: Oxytocin shapes the neural circuitry of trust and trust adaptation in humans. Neuron 2008, 58:639-650

89. Petrovic $P$, Kalisch $R$, Singer T, Dolan RJ: Oxytocin attenuates affective evaluations of conditioned faces and amygdala activity. J Neurosci 2008 28:6607-6615.

90. Kirsch P, Esslinger C, Chen Q, Mier D, Lis S, Siddhanti S, Gruppe H, Mattay VS, Gallhofer B, Meyer-Lindenberg A: Oxytocin modulates neural circuitry for social cognition and fear in humans. J Neurosci 2005, 25:11489-11493.

91. Domes G, Heinrichs M, Glascher J, Buchel C, Braus DF, Herpertz SC: Oxytocin attenuates amygdala responses to emotional faces regardless of valence. Biol Psychiatry 2007, 62:1187-1190.

92. Chakrabarti B, Baron-Cohen S: Variation in the human cannabinoid receptor CNR1 gene modulates gaze duration for happy faces. Molecular Autism 2011, 2:10.

93. Betancur C: Etiological heterogeneity in autism spectrum disorders: more than 100 genetic and genomic disorders and still counting. Brain Res 2011, 1380:42-77.

94. Bill BR, Geschwind DH: Genetic advances in autism: heterogeneity and convergence on shared pathways. Curr Opin Genet Dev 2009, 19:271-278.

95. Green AE, Munafò MR, DeYoung CG, Fossella JA, Fan J, Gray JR: Using genetic data in cognitive neuroscience: from growing pains to genuine insights. Nat Rev Neurosci 2008, 9:710-720.

96. Dichter GS, Damiano CR, Allen JA: Reward circuitry dysfunction in psychiatric and neurodevelopmental disorders and genetic syndromes: animal models and clinical findings. J Neurodev Disord 2012, 4:1-43.

97. Bartz J, Zaki J, Bolger N, Ochsner KN: Social effects of oxytocin in humans: context and person matter. Trends Cogn Sci 2011, 15:301-309.

98. Heinrichs M, Domes G: Neuropeptides and social behaviour: effects of oxytocin and vasopressin in humans. Prog Brain Res 2008, 170:337-350.

99. Hart $A B$, de Wit H, Palmer AA: Candidate gene studies of a promising intermediate phenotype: failure to replicate. Neuropsychopharmacology 2012, 38:802-816. 
100. McCarthy MI, Abecasis GR, Cardon LR, Goldstein DB, Little J, loannidis JPA, Hirschhorn JN: Genome-wide association studies for complex traits: consensus, uncertainty and challenges. Nat Rev Genet 2008, 9:356-369.

101. Bakermans-Kranenburg MJ, van ljzendoorn MH: A sociability gene? Meta-analysis of oxytocin receptor genotype effects in humans. Psychiatr Genet 2013.

102. Bigos KL, Weinberger DR: Imaging genetics - days of future past. Neuroimage 2010, 53:804-809.

103. Knutson B, Greer SM: Anticipatory affect: neural correlates and consequences for choice. Phil Trans Roy Soc B: Biol Sci 2008, 363:3771-3786.

104. Taylor SE: Tend and befriend biobehavioral bases of affiliation under stress. Curr Dir Psychol Sci 2006, 15:273-277.

105. Tomizawa K, Iga N, Lu Y-F, Moriwaki A, Matsushita M, Li S-T, Miyamoto O, Itano T, Matsui H: Oxytocin improves long-lasting spatial memory during motherhood through MAP kinase cascade. Nat Neurosci 2003, 6:384-390.

doi:10.1186/2040-2392-5-7

Cite this article as: Damiano et al: Association between the oxytocin receptor (OXTR) gene and mesolimbic responses to rewards. Molecular Autism 2014 5:7.

\section{Submit your next manuscript to BioMed Central and take full advantage of:}

- Convenient online submission

- Thorough peer review

- No space constraints or color figure charges

- Immediate publication on acceptance

- Inclusion in PubMed, CAS, Scopus and Google Scholar

- Research which is freely available for redistribution 